\title{
New Cosmological Solutions of a Nonlocal Gravity Model
}

\author{
Ivan Dimitrijevic ${ }^{1}$, Branko Dragovich ${ }^{2,3, *}$, Zoran Rakic ${ }^{1} \mathbb{D}$ and Jelena Stankovic ${ }^{4}$ \\ 1 Faculty of Mathematics, University of Belgrade, Studentski Trg 16, 11158 Belgrade, Serbia; \\ ivand@matf.bg.ac.rs (I.D.); zrakic@matf.bg.ac.rs (Z.R.) \\ 2 Institute of Physics, University of Belgrade, 11080 Belgrade, Serbia \\ 3 Mathematical Institute of the Serbian Academy of Sciences and Arts, 11000 Belgrade, Serbia \\ 4 Teacher Education Faculty, University of Belgrade, Kraljice Natalije 43, 11000 Belgrade, Serbia; \\ jelena.grujic@uf.bg.ac.rs \\ * Correspondence: dragovich@ipb.ac.rs
}

Citation: Dimitrijevic, I.; Dragovich,

B.; Rakic, Z.; Stankovic, J. New

Cosmological Solutions of a Nonlocal

Gravity Model. Symmetry 2022, 14, 3.

https: / / doi.org/10.3390/

sym 14010003

Academic Editors: Sergey Vernov and

Vasilis K. Oikonomou

Received: 14 November 2021

Accepted: 16 December 2021

Published: 21 December 2021

Publisher's Note: MDPI stays neutral with regard to jurisdictional claims in published maps and institutional affiliations.

Copyright: (c) 2021 by the authors. Licensee MDPI, Basel, Switzerland. This article is an open access article distributed under the terms and conditions of the Creative Commons Attribution (CC BY) license (https:// creativecommons.org/licenses/by/ $4.0 /)$.

\begin{abstract}
A nonlocal gravity model (2) was introduced and considered recently, and two exact cosmological solutions in flat space were presented. The first solution is related to some radiation effects generated by nonlocal dynamics on dark energy background, while the second one is a nonsingular time symmetric bounce. In the present paper, we investigate other possible exact cosmological solutions and find some the new ones in nonflat space. Used nonlocal gravity dynamics can change the background topology. To solve the corresponding equations of motion, we first look for a solution of the eigenvalue problem $\square(R-4 \Lambda)=q(R-4 \Lambda)$. We also discuss possible extension of this model with a nonlocal operator, symmetric under $\square \longleftrightarrow \square^{-1}$, and its connection with another interesting nonlocal gravity model.
\end{abstract}

Keywords: nonlocal gravity; cosmological solutions; nonsingular bounce; cosmological constant; cosmic radiation; dark energy; dynamical change of topology

\section{Introduction}

The current standard model of cosmology (SMC) [1], also known as the $\Lambda$ CDM model, assumes general relativity (GR) [2] as the theory of the gravitational interaction at all cosmic space-time scales-galactic and cosmological. According to this model, at the current cosmic time, the universe approximately contains $68 \%$ of dark energy (DE), $27 \%$ of dark matter (DM) and $5 \%$ of visible matter. By the $\Lambda$ CDM model, dark matter is responsible for observational dynamics inside and between galaxies, while dark energy causes accelerated expansion of the universe. The $\Lambda \mathrm{CDM}$ model also asserts that DE corresponds to the cosmological constant and that DM is in a cold state. In the last few decades many efforts were made to confirm the existence of DM and DE in the sky or in the laboratory experiments, but they were undiscovered, and their existence still remains hypothetical. A brief review of the recent investigations of DM and DE is presented in [3].

Due to its significant phenomenological achievements and beautiful theoretical properties, GR is considered one of the basic modern physical theories [4]. For example, GR describes the dynamics of the solar system very well. Many important phenomena were also predicted and observationally confirmed: deflection of light near the Sun, black holes, as well as gravitational light redshift, lensing, and waves. However, GR as a theory of gravitation has not been verified at the galactic and cosmological scales. Despite remarkable successes, GR solutions for the black holes and the beginning of the universe contain singularities. In addition, from quantization point of view, GR is a nonrenormalizable theory. Note also that every other physical theory has its domain of validity, which is usually constrained by the space-time scale, complexity of the system under consideration, or by some parameters. There is no a priori reason that GR is an exception and should be theory of gravitation from the Planck scale to the universe as a whole. Taking into account 
all these remarks, it follows that general relativity is not a final gravitational theory and that investigation of its extension is needed; see [5-12] and the references therein.

Since a new physical principle that could say in which direction to extend GR has not been invented so far, there are many approaches to its modification (for a review, see [5-9]). One of the current and attractive approaches to general relativity modification is its nonlocal extension; see, for example, [13-33]. The idea behind nonlocality is that the dynamics of the gravitational field may depend not only on its first and second space-time derivatives, but also on all higher derivatives. It means that the Einstein-Hilbert action should be extended by an additional nonlocal term that contains the d'Alembert-Beltrami operator $\square$ which is mainly employed in two ways: (i) using an analytic expansion $F(\square)=\sum_{n=0}^{+\infty} f_{n} \square^{n}$, or (ii) including in some manner operator $\square^{-1}[13-15,34]$, and its higher powers.

The modification of type (i) comes from ordinary and $p$-adic string theory, see [35] and the references therein. This type of nonlocality improves quantum renormalizability [36-38]. Nonlocal gravity models of type (i) that have attracted much attention are given by action

$$
S=\frac{1}{16 \pi G} \int_{\mathcal{M}} \sqrt{-g}(R-2 \Lambda+P(R) \mathcal{F}(\square) Q(R)) d^{4} x
$$

where $\mathcal{M}$ is a four-dimensional pseudo-Riemannian manifold of signature $(-,+,+,+)$ with metric $\left(g_{\mu \nu}\right), P(R)$ and $Q(R)$ are some differentiable functions of scalar curvature $R, \Lambda$ is the cosmological constant, and $\mathcal{F}(\square)=\sum_{n=0}^{+\infty} f_{n} \square^{n}$. To better see the effects of nonlocal modification of GR in its geometrical sector, action (1) intentionally does not contain the matter term. Derivation of equations of motion that are related to nonlocal gravity (1) is a difficult task, and for details we refer to our paper [39]; see also [20].

Action (1) is rather general and contains several simple nonlocal extensions of GR. $P(R)=Q(R)=R$ is a case that attracted the most attention; see [16,17,24,25,40-47]. It includes also nonlocal extension of the Starobinsky $R^{2}$ inflation model $[28,29]$. This kind of nonlocal investigation started in [16,17] and is an attempt to find a nonsingular bouncing solution of the singularity problem in standard cosmology. It is worth mentioning an interesting model when $P(R)=Q(R)=\sqrt{R-2 \Lambda}$, which contains a cosmological solution $a(t)=A t^{\frac{2}{3}} e^{\frac{\Lambda}{14} t^{2}}$ that mimics an interference between dark matter $\left(t^{\frac{2}{3}}\right)$ and dark energy $\left(e^{\frac{\Lambda}{14} t^{2}}, \Lambda>0\right)$ in flat space $(k=0)$. The explored cosmological parameters are in good agreement with the $\Lambda$ CDM data; see [48].

This paper is devoted to the further investigation of the nonlocal gravity model, which is given by $P(R)=Q(R)=R-4 \Lambda$, and presented in [49]. The nonlocal term $(R-4 \Lambda) \mathcal{F}(\square)(R-4 \Lambda)$ appears as a generalization of $R \mathcal{F}(\square) R$. This model is also of interest as the limit case of model $P(R)=Q(R)=\sqrt{R-2 \Lambda}$ for $|R| \ll|2 \Lambda|$; see Section 2 . In the paper [49], we investigated the exact cosmological solutions for $\Lambda \neq 0, k=0$ : $a_{1}(t)=A \sqrt{t} e^{\frac{\Lambda}{4} t^{2}}$, and $a_{2}(t)=A e^{\Lambda t^{2}}$. The first solution mimics an interplay between dark energy and radiation. The second solution is a nonsingular bounce one and an even function of cosmic time. In this paper, we consider new cosmological solutions with scale factors of two forms: $a(t)=\left(\alpha e^{\lambda t}+\beta e^{-\lambda t}\right)^{\gamma}$ and $a(t)=(\alpha \cos \lambda t+\beta \sin \lambda t)^{\gamma}$, where $\gamma$ is an arbitrary real parameter.

The paper is organized as follows. In Section 2, the concrete nonlocal gravity model is set up and some general properties of the relevant equations of motion are presented. Section 3 contains consideration of various aspects of the corresponding cosmological solutions: a brief review of the two previous results, relevant eigenvalue problem and detailed analysis related to the finding of the new exact cosmological solutions. Discussion and conclusions are presented in Section 4.

\section{Gravity Model with Additional Nonlocal Term $(R-4 \Lambda) \mathcal{F}(\square)(R-4 \Lambda)$}

Recall that the first model of form (1), where $P(R)=Q(R)=R$ and $\Lambda=0$, was considered in [16]. The next nonlocal model [17] of type (1) had also $P(R)=Q(R)=R$, but $\Lambda \neq 0$. Note that in [16,17], as well as in some other models (e.g., see [24,41]), a 
nonlocal operator is $\mathcal{F}(\square)=\sum_{n=0}^{+\infty} f_{n} \square^{n}$, where $f_{0} \neq 0$. Hence these nonlocal gravity models contain a local $R^{2}$ term and present a nonlocal extension of $R^{2}$ gravity; see also nonlocal $R^{2}$ inflation $[28,29]$. What we will consider does not contain local $R^{2}$ term, i.e., $f_{0}=0$.

Nonlocal gravity model under consideration is given by action [49]

$$
S=\frac{1}{16 \pi G} \int d^{4} x \sqrt{-g}(R-2 \Lambda+(R-4 \Lambda) \mathcal{F}(\square)(R-4 \Lambda)),
$$

where $\square=\nabla_{\mu} \nabla^{\mu}=\frac{1}{\sqrt{-g}} \partial_{\mu}\left(\sqrt{-g} g^{\mu v} \partial_{\nu}\right)$ is the d'Alembert-Beltrami operator on the corresponding gravity background and $\mathcal{F}(\square)=\sum_{n=1}^{+\infty} f_{n} \square^{n}$ is the nonlocal operator with all higher order space-time derivatives. Formally, (2) is obtained from (1) taking $P(R)=Q(R)=R-4 \Lambda$ and $f_{0}=0$. However, (2) can be also derived from action

$$
S=\frac{1}{16 \pi G} \int d^{4} x \sqrt{-g}(R-2 \Lambda+\sqrt{R-2 \Lambda} \mathcal{F}(\square) \sqrt{R-2 \Lambda})
$$

which also belongs to the class of nonlocal models (1). In fact, let us start from action (3) and consider the expansion of $\sqrt{R-2 \Lambda}=\sqrt{-2 \Lambda} \sqrt{1-\frac{R}{2 \Lambda}}$ in powers of $\frac{R}{2 \Lambda}$, where $|R| \ll|2 \Lambda|$. Then let us take approximation linear in $\frac{R}{2 \Lambda}$, i.e., one obtains $\sqrt{R-2 \Lambda} \simeq \sqrt{-2 \Lambda}\left(1-\frac{R}{4 \Lambda}\right)$. By this way, the nonlocal term in (3) becomes

$$
\sqrt{R-2 \Lambda} \mathcal{F}(\square) \sqrt{R-2 \Lambda} \simeq-\frac{1}{8 \Lambda}(R-4 \Lambda) \mathcal{F}(\square)(R-4 \Lambda),
$$

where factor $-\frac{1}{8 \Lambda}$ can be included in nonlocal operator $\mathcal{F}(\square)$ by its redefinition. At the same time, the first term $R-2 \Lambda=\sqrt{R-2 \Lambda} \sqrt{R-2 \Lambda}$ remains unchanged in the linear approximation.

As it is already mentioned in Introduction, the nonlocal gravity model (3) is very interesting and promising. It is a natural nonlocal generalization of the de Sitter model

$$
S_{0}=\frac{1}{16 \pi G} \int d^{4} x \sqrt{-g}(R-2 \Lambda),
$$

where generalization is obtained in the following way:

$$
R-2 \Lambda=\sqrt{R-2 \Lambda} \sqrt{R-2 \Lambda} \rightarrow \sqrt{R-2 \Lambda} F(\square) \sqrt{R-2 \Lambda} .
$$

The nonlocal operator $F(\square)$ in (6) is $F(\square)=1+\mathcal{F}(\square)=1+\sum_{n=1}^{+\infty} f_{n} \square^{n}$. The nonlocal de Sitter model (also called nonlocal square root gravity [48]) (3) contains two exact scale factors:

$$
a(t)=A t^{\frac{2}{3}} e^{\frac{\Lambda}{14} t^{2}}, \quad a(t)=A e^{\frac{\Lambda}{6} t^{2}}, \quad k=0 .
$$

The first solution in (7) mimics an interference between dark matter expansion $\left(t^{\frac{2}{3}}\right)$ and dark energy acceleration $\left(e^{\frac{\Lambda}{4} t^{2}}, \Lambda>0\right)$ in flat space $(k=0)$, and the calculated cosmological quantities are in good agreement with the standard model of cosmology; see details in [48]. The second solution in (7) is an example of nonsingular bounce at cosmic time $t=0$.

\section{Equations of Motion}

The next step in the investigation of nonlocal gravity model (2) is finding the corresponding equations of motion (EOM). It is done for a class of models (1) that contain (2); the derivation is presented in [39].

According to [39], the EOM for nonlocal gravity model (1) has the following form:

$$
\hat{G}_{\mu v}=G_{\mu \nu}+\Lambda g_{\mu \nu}-\frac{1}{2} g_{\mu \nu} P(R) \mathcal{F}(\square) Q(R)+R_{\mu \nu} W-K_{\mu \nu} W+\frac{1}{2} \Omega_{\mu v}=0,
$$


where

$$
\begin{aligned}
& W=P^{\prime}(R) \mathcal{F}(\square) Q(R)+Q^{\prime}(R) \mathcal{F}(\square) P(R), \quad K_{\mu \nu}=\nabla_{\mu} \nabla_{v}-g_{\mu v} \square, \\
& \Omega_{\mu v}=\sum_{n=1}^{+\infty} f_{n} \sum_{\ell=0}^{n-1} S_{\mu v}\left(\square^{\ell} P(R), \square^{n-1-\ell} Q(R)\right), \\
& S_{\mu \nu}(A, B)=g_{\mu v}\left(\nabla^{\alpha} A \nabla_{\alpha} B+A \square B\right)-2 \nabla_{\mu} A \nabla_{\nu} B,
\end{aligned}
$$

and $P^{\prime}$ and $Q^{\prime}$ denote the derivatives of $P$ and $Q$ with respect to $R$.

It is clear that EOM (8) are very complicated, comparing them to their local (Einstein) counterpart $G_{\mu v}+\Lambda g_{\mu \nu}=0$. Finding any solutions of (8) is not an easy task. However, in the sequel of this article, we will see how one can find some exact cosmological solutions when $P(R)=Q(R)=R-4 \Lambda$, i.e., in the nonlocal gravity model (2).

First, let us consider the case when $Q(R)=P(R)$. Then, EOM (8) reduce to

$$
\begin{gathered}
G_{\mu v}+\Lambda g_{\mu v}-\frac{g_{\mu v}}{2} P(R) \mathcal{F}(\square) P(R)+R_{\mu v} W-K_{\mu \nu} W+\frac{1}{2} \Omega_{\mu v}=0, \\
W=2 P^{\prime}(R) \mathcal{F}(\square) P(R), \quad K_{\mu v}=\nabla_{\mu} \nabla_{v}-g_{\mu v} \square, \\
\Omega_{\mu v}=\sum_{n=1}^{+\infty} f_{n} \sum_{\ell=0}^{n-1} S_{\mu v}\left(\square^{\ell} P, \square^{n-1-\ell} P\right), \\
S_{\mu v}\left(\square^{\ell} P, \square^{n-1-\ell} P\right)=\sum_{\ell=0}^{n-1}\left(g_{\mu v}\left(\nabla^{\alpha} \square^{\ell} P(R) \nabla_{\alpha} \square^{n-1-\ell} P(R)+\square^{\ell} P(R) \square^{n-\ell} P(R)\right)\right. \\
\left.-2 \nabla_{\mu} \square^{\ell} P(R) \nabla_{v} \square^{n-1-\ell} P(R)\right) .
\end{gathered}
$$

The further significant simplification of EOM can be obtained if $P(R)$ is an eigenfunction of the corresponding d'Alembert-Beltrami operator $\square$, i.e., if the following holds:

$$
\square P(R)=q P(R), \quad \mathcal{F}(\square) P(R)=\mathcal{F}(q) P(R),
$$

where $q=\zeta \Lambda$ ( $\zeta$ dimensionless parameter) is an eigenvalue. Note that parameter $q$ must have the same dimensionality as $\square$, where the dimension of $\square$ is $T^{-2}$ in natural units $(\bar{h}=c=1)$. Hence, $q$ has to be proportional to $\Lambda$ since there is only the cosmological constant $\Lambda$ in the above EOM with dimension as $\square$. Moreover, $q=\zeta \Lambda$ naturally appears in all concrete cases and there is no need for a new constant in this nonlocal gravity model without matter. Then

$$
\begin{gathered}
W=2 \mathcal{F}(q) P^{\prime} P, \quad \mathcal{F}(q)=\sum_{n=1}^{+\infty} f_{n} q^{n}, \\
\Omega_{\mu v}=\mathcal{F}^{\prime}(q) S_{\mu v}(P, P), \\
G_{\mu v}+\Lambda g_{\mu v}+\mathcal{F}(q)\left(2\left(R_{\mu v}-K_{\mu v}\right) P P^{\prime}-\frac{g_{\mu v}}{2} P^{2}\right) \\
+\frac{1}{2} \mathcal{F}^{\prime}(q) S_{\mu v}(P, P)=0 .
\end{gathered}
$$

Both expressions in (17) are evident. Equality (18) is obtained as follows:

$$
\begin{aligned}
\Omega_{\mu v} & =\sum_{n=1}^{\infty} f_{n} \sum_{\ell=0}^{n-1} S_{\mu v}\left(\square^{\ell} P, \square^{n-1-\ell} P\right)=\sum_{n=1}^{\infty} f_{n} \sum_{\ell=0}^{n-1} q^{n-1} S_{\mu v}(P, P) \\
& =\sum_{n=1}^{\infty} f_{n} n q^{n-1} S_{\mu v}(P, P)=\mathcal{F}^{\prime}(q) S_{\mu v}(P, P) .
\end{aligned}
$$


Finally, take $P=R-4 \Lambda$. Then $P P^{\prime}=P=R-4 \Lambda$ and EOM become

$$
\begin{aligned}
& G_{\mu \nu}+\Lambda g_{\mu \nu}+\mathcal{F}(q)\left(G_{\mu v}+R_{\mu \nu}-2 \nabla_{\mu} \nabla_{\nu}+2 g_{\mu \nu}(\Lambda+q)\right)(R-4 \Lambda) \\
& +\frac{1}{2} \mathcal{F}^{\prime}(q) S_{\mu \nu}(R-4 \Lambda, R-4 \Lambda)=0 .
\end{aligned}
$$

In some cases, there is solution when $\mathcal{F}^{\prime}(q)=0$, and then problem (21) reduces to

$$
\begin{aligned}
& \mathcal{F}^{\prime}(q)=0, \text { and } \\
& G_{\mu v}+\Lambda g_{\mu v}+\mathcal{F}(q)\left(G_{\mu v}+R_{\mu \nu}-2 \nabla_{\mu} \nabla_{v}+2 g_{\mu v}(\Lambda+q)\right)(R-4 \Lambda)=0 .
\end{aligned}
$$

In finding the cosmological solutions, we start from Equation (21).

\section{Cosmological Solutions}

In this section, we are mainly interested in the finding and investigation of some new exact cosmological solutions of nonlocal gravity model (2).

Since the universe is homogeneous and isotropic at large cosmic scales, its evolution satisfies the Friedmann-Lemaître-Robertson-Walker (FLRW) metric

$$
d s^{2}=-d t^{2}+a^{2}(t)\left(\frac{d r^{2}}{1-k r^{2}}+r^{2} d \theta^{2}+r^{2} \sin ^{2} \theta d \phi^{2}\right), \quad(c=1), k=0, \pm 1,
$$

where $a(t)$ is the cosmic scale factor that contains information on expansion (or contraction) and $k$ is the constant curvature parameter.

The d'Alembert-Beltrami operator $\square$, the Hubble parameter $H$ and the Ricci scalar $R$ for the FLRW metric are

$$
\begin{aligned}
& \square=-\frac{\partial^{2}}{\partial t^{2}}-3 H(t) \frac{\partial}{\partial t^{\prime}} \quad H(t)=\frac{\dot{a}}{a} \\
& R(t)=6\left(\frac{\ddot{a}}{a}+\left(\frac{\dot{a}}{a}\right)^{2}+\frac{k}{a^{2}}\right), \quad \dot{a}=\frac{\partial a}{\partial t} .
\end{aligned}
$$

Since the universe is homogeneous and isotropic, there are only two independent equations of motion (21). It is convenient to use the trace and 00-component of (21):

$$
\begin{aligned}
& (R-4 \Lambda)[\mathcal{F}(\zeta \Lambda)(8+6 \zeta) \Lambda-1]+\frac{1}{2} \mathcal{F}^{\prime}(\zeta \Lambda) S(R-4 \Lambda, R-4 \Lambda)=0, \\
& G_{00}-\Lambda+\mathcal{F}(\zeta \Lambda)\left(2 R_{00}+\frac{1}{2} R-2 \partial_{0}^{2}-2(1+\zeta) \Lambda\right)(R-4 \Lambda) \\
& +\frac{1}{2} \mathcal{F}^{\prime}(\zeta \Lambda) S_{00}(R-4 \Lambda, R-4 \Lambda)=0,
\end{aligned}
$$

where $S(R-4 \Lambda, R-4 \Lambda)=g^{\mu v} S_{\mu v}(R-4 \Lambda, R-4 \Lambda)$ and equality $\square(R-4 \Lambda)=q(R-$ $4 \Lambda)=\zeta \Lambda(R-4 \Lambda)$ is taken into account. According to (27), we have to use

$$
R_{00}=-3 \frac{\ddot{a}}{a}, \quad G_{00}=3 \frac{\dot{a}^{2}+k}{a^{2}} .
$$

Note that EOM (21) can be rewritten in the form of general relativity

$$
\hat{G}_{\mu v}=G_{\mu v}+\Lambda g_{\mu \nu}-8 \pi G \hat{T}_{\mu \nu}=0, \quad \nabla^{\mu} \hat{G}_{\mu v}=0,
$$

where $\hat{T}_{\mu \nu}$ is the corresponding effective energy-momentum tensor. The related Friedmann equations to (29) are

$$
\frac{\ddot{a}}{a}=-\frac{4 \pi G}{3}(\bar{\rho}+3 \bar{p})+\frac{\Lambda}{3}, \quad \frac{\dot{a}^{2}+k}{a^{2}}=\frac{8 \pi G}{3} \bar{\rho}+\frac{\Lambda}{3},
$$


where $\bar{\rho}$ is an effective energy density and $\bar{p}$ is an effective pressure of the universe. The corresponding equation of state is

$$
\bar{p}(t)=\bar{w}(t) \bar{\rho}(t)
$$

where $\bar{w}(t)$ is a dimensionless parameter that may depend on time.

It is worth noting that the Minkowski space $(a(t)=$ const., $R=\Lambda=k=0)$ is also a solution of EOM (26) and (27).

\subsection{Two Previous Exact Solutions}

In order to have more complete insight into $a(t)$ solutions of nonlocal model (2), we want to first briefly review the previously found two nontrivial solutions [49], and after that present new exact solutions.

We found the following exact cosmological solutions, $\Lambda \neq 0, k=0$ :

$$
\begin{array}{ll}
a_{1}(t)=A t^{\frac{1}{2}} e^{\frac{\Lambda}{4} t^{2},} & \square(R-4 \Lambda)=-3 \Lambda(R-4 \Lambda), \\
a_{2}(t)=A e^{\Lambda t^{2}}, & \square(R-4 \Lambda)=-12 \Lambda(R-4 \Lambda) .
\end{array}
$$

We explicitly found expression for $R(t), H(t)$, solved the corresponding eigenvalue problems and EOM for both $a_{1}(t)$ and $a_{2}(t)$, and also found constraints on the nonlocal operator function $\mathcal{F}(\square)$ :

$$
\begin{array}{ll}
\left(a_{1}\right): \mathcal{F}(-3 \Lambda)=-\frac{1}{10 \Lambda}, \quad \mathcal{F}^{\prime}(-3 \Lambda)=0, & \Lambda \neq 0 \\
\left(a_{2}\right): & \mathcal{F}(-12 \Lambda)=-\frac{1}{64 \Lambda}, \quad \mathcal{F}^{\prime}(-12 \Lambda)=0, \quad \Lambda \neq 0
\end{array}
$$

that are simply satisfied by

$$
\begin{aligned}
& \left(a_{1}\right): \mathcal{F}(\square)=\frac{\square}{30 \Lambda^{2}} \exp \left(\frac{\square}{3 \Lambda}+1\right), \\
& \left(a_{2}\right): \mathcal{F}(\square)=\frac{\square}{768 \Lambda^{2}} \exp \left(\frac{\square}{12 \Lambda}+1\right),
\end{aligned}
$$

respectively.

The solution of the effective Friedmann equations were also found in both cases, and consequently, the equations of state are:

$$
\begin{aligned}
& \left(a_{1}\right): \quad \bar{w}=\frac{\bar{p}(t)}{\bar{\rho}(t)}=\frac{1-6 \Lambda t^{2}-3 \Lambda^{2} t^{4}}{3+2 \Lambda t^{2}+3 \Lambda^{2} t^{4}} \rightarrow \begin{cases}-1, & t \rightarrow \infty \\
\frac{1}{3}, & t \rightarrow 0 .\end{cases} \\
& \left(a_{2}\right): \bar{w}=\frac{-12 \Lambda t^{2}-3}{12 \Lambda t^{2}-1} \rightarrow \begin{cases}-1, & t \rightarrow \infty \\
3, & t \rightarrow 0 .\end{cases}
\end{aligned}
$$

$\left(a_{1}\right)$ : This solution may be relevant to the early radiation dominant universe and to its late accelerated expansion. The solution mimics interference between expansion with radiation $a(t)=A \sqrt{t}$ and a dark energy $a(t)=A e^{\frac{\Lambda}{4}} t^{2}, \Lambda>0$.

$\left(a_{2}\right)$ : The solution $a_{2}(t)=A e^{\Lambda t^{2}}$ is an even function of cosmic time and presents an example of the nonsingular bounce.

We now explore the existence of new cosmological solutions with scale factors $a(t)$ similar to those well known in the de Sitter local model (5), but with time-dependent scalar curvature $R(t)$ so that $\square(R-4 \Lambda)=q(R-4 \Lambda)$, where $q \neq 0$. In fact, scale factors in the 
form $a(t)=\left(\alpha e^{\lambda t}+\beta e^{-\lambda t}\right)^{\gamma}$ and $a(t)=(\alpha \cos \lambda t+\beta \sin \lambda t)^{\gamma}$ are investigated. We see that such solutions exist, and their significance is discussed in Section 4.

\subsection{Eigenvalue Problem for New Cosmological Solutions}

Let us consider the scale factor

$$
a(t)=\left(\alpha e^{\lambda t}+\beta e^{-\lambda t}\right)^{\gamma},
$$

and an eigenvalue problem

$$
\square(R-4 \Lambda)=q(R-4 \Lambda)=\zeta \Lambda(R-4 \Lambda),
$$

for a dimensionless constant $\zeta$ that is determined later. Equality (41) can be expanded into

$$
\begin{aligned}
& \left(\beta+\alpha e^{2 \lambda t}\right)^{2}\left(A_{0}+A_{1} e^{2 \lambda t}+A_{2} e^{4 \lambda t}\right) \\
& +2\left(\alpha e^{\lambda t}+\beta e^{-\lambda t}\right)^{2 \gamma}\left(B_{0}+B_{1} e^{2 \lambda t}+B_{2} e^{4 \lambda t}+B_{3} e^{6 \lambda t}+B_{4} e^{8 \lambda t}\right)=0
\end{aligned}
$$

where

$$
\begin{aligned}
& A_{0}=3 k \beta^{2}\left(q-2 \gamma^{2} \lambda^{2}\right), \\
& A_{1}=6 k \alpha \beta\left(2(\gamma-2) \gamma \lambda^{2}+q\right), \\
& A_{2}=3 k \alpha^{2}\left(q-2 \gamma^{2} \lambda^{2}\right),
\end{aligned}
$$

and

$$
\begin{aligned}
& B_{0}=\beta^{4} q\left(3 \gamma^{2} \lambda^{2}-\Lambda\right), \\
& B_{1}=2 \alpha \beta^{3}\left(6 \gamma\left(6 \gamma^{2}-7 \gamma+2\right) \lambda^{4}+q\left(3 \gamma \lambda^{2}-2 \Lambda\right)\right), \\
& B_{2}=-6 \alpha^{2} \beta^{2}\left(4 \gamma\left(6 \gamma^{2}-11 \gamma+4\right) \lambda^{4}+q\left(\gamma^{2} \lambda^{2}-2 \gamma \lambda^{2}+\Lambda\right)\right), \\
& B_{3}=2 \alpha^{3} \beta\left(6 \gamma\left(6 \gamma^{2}-7 \gamma+2\right) \lambda^{4}+q\left(3 \gamma \lambda^{2}-2 \Lambda\right)\right), \\
& B_{4}=\alpha^{4} q\left(3 \gamma^{2} \lambda^{2}-\Lambda\right) .
\end{aligned}
$$

In the case $\alpha \beta=0$, i.e., $\alpha=0$ or $\beta=0$, the eigenvalue problem $\square(R-4 \Lambda)=$ $q(R-4 \Lambda)$ has a nontrivial solution in the following two cases:

1. $k=0, \Lambda=3 \gamma^{2} \lambda^{2}$

2. $k \neq 0, q=2 \gamma^{2} \lambda^{2}, \Lambda=3 \gamma^{2} \lambda^{2}$.

When $\alpha \beta \neq 0$, then functions $e^{2 \lambda t}$ and $\left(\alpha e^{\lambda t}+\beta e^{-\lambda t}\right)^{2 \gamma}$ are linearly independent. In this case, we can split Equation (42) into

$$
A_{0}=A_{1}=A_{2}=0, \quad B_{0}=B_{1}=B_{2}=B_{3}=B_{4}=0 .
$$

The previous Equations (45) are satisfied in the following two cases:

$$
\begin{aligned}
& \text { 1. } \gamma=1, q=2 \lambda^{2}, \Lambda=3 \lambda^{2}, k \in\{0,-1,1\}, \\
& \text { 2. } \gamma=\frac{1}{2}, \Lambda=\frac{3}{4} \lambda^{2}, k=0 .
\end{aligned}
$$

Hence, the only two possibilities for parameter $\gamma$ are $\gamma=1$ and $\gamma=\frac{1}{2}$.

Now, let us consider the scale factor

$$
a(t)=(\alpha \cos \lambda t+\beta \sin \lambda t)^{\gamma},
$$


and the corresponding eigenvalue problem

$$
\square(R-4 \Lambda)=q(R-4 \Lambda) .
$$

Similarly as in the previous case, if we replace $\lambda$ by $\mathrm{i} \lambda$ in the scale factor $a(t)=\left(\alpha e^{\lambda t}+\beta e^{-\lambda t}\right)^{\gamma}$, we obtain that the eigenvalue problem (49) has solution in the following two cases:

$$
\begin{aligned}
& \text { 1. } \gamma=1, q=-2 \lambda^{2}, \Lambda=-3 \lambda^{2}, k \in\{0,-1,1\}, \\
& \text { 2. } \gamma=\frac{1}{2}, \Lambda=-\frac{3}{4} \lambda^{2}, k=0 .
\end{aligned}
$$

As result of the solution of eigenvalue problem (41), we obtained not only concrete eigenvalue $q$ but also possible values of $\gamma$ and $\lambda$ for the cosmic scale factor of the form (40) and (48). In fact, we found that nonlocal gravity model (2) may have the following new cosmological solutions:

$$
\begin{aligned}
& a_{3}(t)=\alpha e^{\sqrt{\frac{1}{3} \Lambda} t}+\beta e^{-\sqrt{\frac{1}{3} \Lambda} t}, \quad \Lambda \geq 0, \\
& a_{4}(t)=\left(\alpha e^{2 \sqrt{\frac{1}{3} \Lambda} t}+\beta e^{-2 \sqrt{\frac{1}{3} \Lambda} t}\right)^{\frac{1}{2}}, \quad \Lambda \geq 0, \\
& a_{5}(t)=\alpha \cos \sqrt{-\frac{1}{3} \Lambda} t+\beta \sin \sqrt{-\frac{1}{3} \Lambda} t, \quad \Lambda \leq 0, \\
& a_{6}(t)=\left(\alpha \cos 2 \sqrt{-\frac{1}{3} \Lambda} t+\beta \sin 2 \sqrt{-\frac{1}{3} \Lambda} t\right)^{\frac{1}{2}}, \quad \Lambda \leq 0 .
\end{aligned}
$$

By additional requirements that scale factors, (52)-(55) satisfy equations of motion (26), and (27) gives the possibility to determine values of $\alpha$ and $\beta$, fix curvature constant $k$ and obtain constraints on $\mathcal{F}(\square)$ and $\mathcal{F}^{\prime}(\square)$. In the following four subsections, we give more details.

3.3. Cosmological Solution of the Form $a_{3}(t)=\alpha e^{\sqrt{\frac{\Lambda}{3}}} t+\beta e^{-\sqrt{\frac{\Lambda}{3}} t}$

In this case, we have

$$
\begin{aligned}
& \dot{a}(t)=\sqrt{\frac{\Lambda}{3}}\left(\alpha e^{\sqrt{\frac{\Lambda}{3}} t}-\beta e^{-\sqrt{\frac{\Lambda}{3}} t}\right), \quad \ddot{a}(t)=\frac{\Lambda}{3} a(t), \\
& R(t)=4 \Lambda+(6 k-8 \Lambda \alpha \beta) a(t)^{-2}, \\
& H(t)=\sqrt{\frac{\Lambda}{3}}\left(1-2 \beta e^{-\sqrt{\frac{\Lambda}{3}} t} a(t)^{-1}\right), \\
& R_{00}=-\Lambda, \quad G_{00}=\Lambda+(3 k-4 \Lambda \alpha \beta) a(t)^{-2} .
\end{aligned}
$$

The corresponding eigenvalue problem has the following solution:

$$
\square(R-4 \Lambda)=\frac{2}{3} \Lambda(R-4 \Lambda), \quad \mathcal{F}(\square)(R-4 \Lambda)=\mathcal{F}\left(\frac{2}{3} \Lambda\right)(R-4 \Lambda) .
$$

Using the solution of eigenvalue problem (60), the trace and 00 component of EOM are

$$
\begin{aligned}
& \left(4 \alpha \beta \frac{\Lambda}{3}-k\right)\left(T_{0}+T_{1} e^{2 \sqrt{\frac{\Lambda}{3}} t}+T_{2} e^{4 \sqrt{\frac{\Lambda}{3}} t}+T_{3} e^{6 \sqrt{\frac{\Lambda}{3}} t}+T_{4} e^{8 \sqrt{\frac{\Lambda}{3}} t}\right)=0, \\
& \left(4 \alpha \beta \frac{\Lambda}{3}-k\right)\left(Z_{0}+Z_{1} e^{2 \sqrt{\frac{\Lambda}{3}} t}+Z_{2} e^{4 \sqrt{\frac{\Lambda}{3}} t}+Z_{3} e^{6 \sqrt{\frac{\Lambda}{3}} t}+Z_{4} e^{8 \sqrt{\frac{\Lambda}{3}} t}\right)=0,
\end{aligned}
$$


where

$$
\begin{aligned}
& T_{0}=\beta^{4}\left(12 \Lambda \mathcal{F}\left(\frac{2}{3} \Lambda\right)-1\right), \\
& T_{1}=4 \alpha \beta^{3}\left(12 \Lambda \mathcal{F}\left(\frac{2}{3} \Lambda\right)-1\right), \\
& T_{2}=-6 \alpha \beta\left(\alpha \beta\left(1-12 \Lambda \mathcal{F}\left(\frac{2}{3} \Lambda\right)\right)-\frac{16}{3} \Lambda \mathcal{F}^{\prime}\left(\frac{2}{3} \Lambda\right)\left(k-4 \alpha \beta \frac{\Lambda}{3}\right)\right), \\
& T_{3}=4 \alpha^{3} \beta\left(12 \Lambda \mathcal{F}\left(\frac{2}{3} \Lambda\right)-1\right), \\
& T_{4}=\alpha^{4}\left(12 \Lambda \mathcal{F}\left(\frac{2}{3} \Lambda\right)-1\right),
\end{aligned}
$$

and

$$
\begin{aligned}
& Z_{0}=\beta^{4}\left(1-12 \Lambda \mathcal{F}\left(\frac{2}{3} \Lambda\right)\right) \\
& Z_{1}=2 \beta^{2}\left(2 \alpha \beta-6 \Lambda \mathcal{F}^{\prime}\left(\frac{2}{3} \Lambda\right)\left(k-4 \alpha \beta \frac{\Lambda}{3}\right)+3 \mathcal{F}\left(\frac{2}{3} \Lambda\right)(k-4 \alpha \beta \Lambda)\right) \\
& Z_{2}=6 \alpha \beta\left(\alpha \beta+\frac{4}{3} \Lambda \mathcal{F}^{\prime}\left(\frac{2}{3} \Lambda\right)\left(k-4 \alpha \beta \frac{\Lambda}{3}\right)+2 \mathcal{F}\left(\frac{2}{3} \Lambda\right)(k-2 \alpha \beta \Lambda)\right) \\
& Z_{3}=2 \alpha^{2}\left(2 \alpha \beta-6 \Lambda \mathcal{F}^{\prime}\left(\frac{2}{3} \Lambda\right)\left(k-4 \alpha \beta \frac{\Lambda}{3}\right)+3 \mathcal{F}\left(\frac{2}{3} \Lambda\right)(k-4 \alpha \beta \Lambda)\right) \\
& Z_{4}=\alpha^{4}\left(1-12 \Lambda \mathcal{F}\left(\frac{2}{3} \Lambda\right)\right)
\end{aligned}
$$

These two equations are polynomials in $e^{2 \sqrt{\frac{\lambda}{3}} t}$. Both equations are clearly satisfied if $\alpha \beta=\frac{3 k}{4 \Lambda}$. On the other hand, if $\alpha \beta \neq \frac{3 k}{4 \Lambda}$, it remains to solve the system of equations

$$
T_{0}=T_{1}=T_{2}=T_{3}=T_{4}=0, \quad Z_{0}=Z_{1}=Z_{2}=Z_{3}=Z_{4}=0 .
$$

Equations of motion are satisfied in the following three nontrivial cases:

$$
\begin{aligned}
& \text { (i) : } \quad \alpha \beta=\frac{3 k}{4 \Lambda}, \\
& \text { (ii) : } \alpha \beta=0, \mathcal{F}\left(\frac{2}{3} \Lambda\right)=\frac{1}{12 \Lambda}, \mathcal{F}^{\prime}\left(\frac{2}{3} \Lambda\right)=\frac{1}{24 \Lambda^{2}}, k \neq 0, \\
& \text { (iii) : } \quad \alpha \beta=-\frac{k}{4 \Lambda}, \mathcal{F}\left(\frac{2}{3} \Lambda\right)=\frac{1}{12 \Lambda}, \mathcal{F}^{\prime}\left(\frac{2}{3} \Lambda\right)=0 .
\end{aligned}
$$

(i): In the first case, we have $R(t)=4 \Lambda$. For $k=0$, we have $\alpha \beta=0$ and consequently, $a(t) \sim e^{ \pm \sqrt{\frac{\Lambda}{3}}} t$. Additionally, since $\Lambda>0, a(t)=\sqrt{\frac{3}{\Lambda}} \cosh \sqrt{\frac{\Lambda}{3}} t$ requires $k=+1$, while $a(t)=\sqrt{\frac{3}{\Lambda}} \sinh \sqrt{\frac{\Lambda}{3}} t$ if $k=-1$.

(ii): In the second case $\alpha=0$ or $\beta=0$. For $\alpha=0$ we have $a(t)=\beta e^{-\sqrt{\frac{\Lambda}{3}} t}$ and $R(t)=6 k a(t)^{-2}+4 \Lambda$. Analogously, for $\beta=0$ we have $a(t)=\alpha e^{\sqrt{\frac{\Lambda}{3}}} t$ and $R(t)=6 k a(t)^{-2}+4 \Lambda$.

(iii): In the third case, we have $R(t)=4 \Lambda+8 k a(t)^{-2}$. If $k=-1$ there is $\varphi$ such that

$$
\alpha+\beta=\frac{1}{\sqrt{\Lambda}} \cosh \varphi, \quad \alpha-\beta=\frac{1}{\sqrt{\Lambda}} \sinh \varphi .
$$


Now, we can transform scale factor $a(t)=\alpha e^{\sqrt{\frac{\Lambda}{3}}} t+\beta e^{-\sqrt{\frac{\Lambda}{3}} t}$ to

$$
a(t)=\frac{1}{\sqrt{\Lambda}} \cosh \left(\varphi+\sqrt{\frac{\Lambda}{3}} t\right), \quad k=-1 .
$$

If $k=+1$, there is such $\varphi$ that

$$
\alpha+\beta=\frac{1}{\sqrt{\Lambda}} \sinh \varphi, \quad \alpha-\beta=\frac{1}{\sqrt{\Lambda}} \cosh \varphi .
$$

Consequently, we can transform scale factor $a(t)=\alpha e^{\sqrt{\frac{\Lambda}{3}} t}+\beta e^{-\sqrt{\frac{\Lambda}{3}}} t$ to

$$
a(t)=\frac{1}{\sqrt{\Lambda}} \sinh \left(\varphi+\sqrt{\frac{\Lambda}{3}} t\right)
$$

The effective energy density and pressure are given by

$$
\bar{\rho}=\frac{3}{8 \pi G}\left(k-\frac{4}{3} \Lambda \alpha \beta\right) a(t)^{-2}, \quad \bar{p}=-\frac{1}{8 \pi G}\left(k-\frac{4}{3} \Lambda \alpha \beta\right) a(t)^{-2} .
$$

For $k \neq \frac{4}{3} \Lambda \alpha \beta$, the corresponding $\bar{w}$ parameter is $\bar{w}=-\frac{1}{3}$.

3.4. Cosmological Solutions of the Form $a_{4}(t)=\left(\alpha e^{2 \sqrt{\frac{\Lambda}{3}} t}+\beta e^{-2 \sqrt{\frac{\Lambda}{3}} t}\right)^{\frac{1}{2}}$

According to solution (47) of the related eigenvalue problem, in this case, $k=0$. The corresponding Ricci scalar is

$$
R=4 \Lambda \text {. }
$$

The EOM yield the condition

$$
\alpha \beta=0
$$

Hence, there are only solutions $a(t) \sim e^{ \pm \sqrt{\frac{\Lambda}{3}}} t$, what is what we have in the Einstein theory of gravity. Since the corresponding eigenvalue is zero, i.e., $\square(R-4 \Lambda)=0$, solutions of the form $a_{4}(t)=\left(\alpha e^{2 \sqrt{\frac{\Lambda}{3}} t}+\beta e^{-2 \sqrt{\frac{\Lambda}{3}} t}\right)^{\frac{1}{2}}$ are trivial at the classical level from the point of view of the nonlocal gravity model under consideration.

3.5. Cosmological Solutions of the Form $a_{5}(t)=\alpha \cos \sqrt{-\frac{\Lambda}{3}} t+\beta \sin \sqrt{-\frac{\Lambda}{3}} t$

In this case, we have

$$
\begin{aligned}
& \dot{a}(t)=\sqrt{-\frac{\Lambda}{3}}\left(\beta \cos \sqrt{-\frac{\Lambda}{3}} t-\alpha \sin \sqrt{-\frac{\Lambda}{3}} t\right), \quad \ddot{a}(t)=\frac{\Lambda}{3} a(t), \\
& R(t)=4 \Lambda+6\left(k-\left(\alpha^{2}+\beta^{2}\right) \frac{\Lambda}{3} a(t)^{-2},\right. \\
& H(t)=\sqrt{-\frac{\Lambda}{3}}\left(\beta \cos \sqrt{-\frac{\Lambda}{3}} t-\alpha \sin \sqrt{-\frac{\Lambda}{3}} t\right) a(t)^{-1}, \\
& R_{00}=-\Lambda \quad G_{00}=3\left(k-\frac{\Lambda}{3}\left(\beta \cos \sqrt{-\frac{\Lambda}{3}} t-\alpha \sin \sqrt{-\frac{\Lambda}{3}} t\right)^{2}\right) a(t)^{-2} .
\end{aligned}
$$

The corresponding eigenvalue problem has the same solution as in the previous case (60), i.e.,

$$
\square(R-4 \Lambda)=\frac{2}{3} \Lambda(R-4 \Lambda), \quad \mathcal{F}(\square)(R-4 \Lambda)=\mathcal{F}\left(\frac{2}{3} \Lambda\right)(R-4 \Lambda) .
$$


Trace and 00 component of equations of motion read

$$
\begin{aligned}
& \left(k-\frac{\Lambda}{3}\left(\alpha^{2}+\beta^{2}\right)\right)\left(U_{0}+U_{1} e^{2 \mathrm{i} \sqrt{-\frac{\Lambda}{3}} t}+U_{2} e^{4 \mathrm{i} \sqrt{-\frac{\Lambda}{3}} t}+U_{3} e^{6 \mathrm{i} \sqrt{-\frac{\Lambda}{3}} t}+U_{4} e^{8 \mathrm{i} \sqrt{-\frac{\Lambda}{3}} t}\right)=0, \\
& \left(k-\frac{\Lambda}{3}\left(\alpha^{2}+\beta^{2}\right)\right)\left(V_{0}+V_{1} e^{2 \mathrm{i} \sqrt{-\frac{\Lambda}{3}} t}+V_{2} e^{4 \mathrm{i} \sqrt{-\frac{\Lambda}{3}} t}+V_{3} e^{6 \mathrm{i} \sqrt{-\frac{\Lambda}{3}} t}+V_{4} e^{8 \mathrm{i} \sqrt{-\frac{\Lambda}{3}} t}\right)=0
\end{aligned}
$$

where

$$
\begin{aligned}
& U_{0}=(\alpha+\mathrm{i} \beta)^{4}\left(1-12 \Lambda \mathcal{F}\left(\frac{2}{3} \Lambda\right)\right) \\
& U_{1}=4(\alpha+\mathrm{i} \beta)^{3}(\alpha-\mathrm{i} \beta)\left(1-12 \Lambda \mathcal{F}\left(\frac{2}{3} \Lambda\right)\right) \\
& U_{2}=6\left(\alpha^{2}+\beta^{2}\right)\left(\left(\alpha^{2}+\beta^{2}\right)\left(1+\frac{64}{9} \Lambda^{2} \mathcal{F}^{\prime}\left(\frac{2}{3} \Lambda\right)-12 \Lambda \mathcal{F}\left(\frac{2}{3} \Lambda\right)\right)-64 k \frac{\Lambda}{3} \mathcal{F}^{\prime}\left(\frac{2}{3} \Lambda\right)\right), \\
& U_{3}=4(\alpha+\mathrm{i} \beta)(\alpha-\mathrm{i} \beta)^{3}\left(1-12 \Lambda \mathcal{F}\left(\frac{2}{3} \Lambda\right)\right) \\
& U_{4}=(\alpha-\mathrm{i} \beta)^{4}\left(1-12 \Lambda \mathcal{F}\left(\frac{2}{3} \Lambda\right)\right)
\end{aligned}
$$

and

$$
\begin{aligned}
V_{0} & =(\alpha+\mathrm{i} \beta)^{4}\left(1-12 \Lambda \mathcal{F}\left(\frac{2}{3} \Lambda\right)\right), \\
V_{1} & =4(\alpha+\mathrm{i} \beta)^{2}\left(\left(\alpha^{2}+\beta^{2}\right)\left(1+4 \Lambda^{2} \mathcal{F}^{\prime}\left(\frac{2}{3} \Lambda\right)-6 \Lambda \mathcal{F}\left(\frac{2}{3} \Lambda\right)\right)\right. \\
& \left.+6 k\left(\mathcal{F}\left(\frac{2}{3} \Lambda\right)-2 \Lambda \mathcal{F}^{\prime}\left(\frac{2}{3} \Lambda\right)\right)\right), \\
V_{2} & =6\left(\alpha^{2}+\beta^{2}\right)\left(\left(\alpha^{2}+\beta^{2}\right)\left(1-\frac{16}{9} \Lambda^{2} \mathcal{F}^{\prime}\left(\frac{2}{3} \Lambda\right)-4 \Lambda \mathcal{F}\left(\frac{2}{3} \Lambda\right)\right)\right. \\
& \left.+8 k\left(\mathcal{F}\left(\frac{2}{3} \Lambda\right)+\frac{2}{3} \Lambda \mathcal{F}^{\prime}\left(\frac{2}{3} \Lambda\right)\right)\right), \\
V_{3} & =4(\alpha-i \beta)^{2}\left(\left(\alpha^{2}+\beta^{2}\right)\left(1+4 \Lambda^{2} \mathcal{F}^{\prime}\left(\frac{2}{3} \Lambda\right)-6 \Lambda \mathcal{F}\left(\frac{2}{3} \Lambda\right)\right)\right. \\
& \left.+6 k\left(\mathcal{F}\left(\frac{2}{3} \Lambda\right)-2 \Lambda \mathcal{F}^{\prime}\left(\frac{2}{3} \Lambda\right)\right)\right), \\
V_{4} & =(\alpha-i \beta)^{4}\left(1-12 \Lambda \mathcal{F}\left(\frac{2}{3} \Lambda\right)\right) .
\end{aligned}
$$

We consider these equations as polynomials in $e^{2 \mathrm{i} \sqrt{-\frac{\Lambda}{3}}} t$. It is clear that the equations are satisfied for $\alpha^{2}+\beta^{2}=\frac{3 k}{\Lambda}$. In the other case, $\alpha^{2}+\beta^{2} \neq \frac{3 k}{\Lambda}$ it remains to solve the following system of equations

$$
U_{0}=U_{1}=U_{2}=U_{3}=U_{4}=0, \quad V_{0}=V_{1}=V_{2}=V_{3}=V_{4}=0 .
$$

Equations of motion are satisfied in the following two nontrivial cases:

$$
\begin{aligned}
& \text { (i) }: \alpha^{2}+\beta^{2}=\frac{3 k}{\Lambda}, \\
& \text { (ii) : } \quad \mathcal{F}\left(\frac{2}{3} \Lambda\right)=\frac{1}{12 \Lambda}, \mathcal{F}^{\prime}\left(\frac{2}{3} \Lambda\right)=0, \alpha^{2}+\beta^{2}=-\frac{k}{\Lambda} .
\end{aligned}
$$

(i): In the first case, we have $R(t)=4 \Lambda$. 
(ii): In the second case, we have $R(t)=4 \Lambda+8 k a(t)^{-2}$. Taking $k=+1$, there exists $\varphi$ such that

$$
\alpha=\frac{1}{\sqrt{-\Lambda}} \sin \varphi, \quad \beta=\frac{1}{\sqrt{-\Lambda}} \cos \varphi
$$

Now, we can transform scale factor $a(t)=\alpha \cos \sqrt{-\frac{\Lambda}{3}} t+\beta \sin \sqrt{-\frac{\Lambda}{3}} t$ to

$$
a(t)=\frac{1}{\sqrt{-\Lambda}} \sin \left(\sqrt{-\frac{\Lambda}{3}} t-\varphi\right) .
$$

The effective energy density and pressure are

$$
\bar{\rho}=\frac{3 k-\Lambda\left(\alpha^{2}+\beta^{2}\right)}{8 \pi G a(t)^{2}}, \quad \bar{p}=\frac{\Lambda\left(\alpha^{2}+\beta^{2}\right)-3 k}{24 \pi G a(t)^{2}} .
$$

For $k \neq \frac{\Lambda}{3}\left(\alpha^{2}+\beta^{2}\right)$ we have $\bar{w}=-\frac{1}{3}$.

3.6. Cosmological Solutions of the Form $a_{6}(t)=\left(\alpha \cos 2 \sqrt{-\frac{\Lambda}{3}} t+\beta \sin 2 \sqrt{-\frac{\Lambda}{3}} t\right)^{\frac{1}{2}}$

In this case,

$$
R=4 \Lambda, \quad k=0 .
$$

From the equations of motion follows

$$
\alpha^{2}+\beta^{2}=0
$$

Hence, there are no nontrivial solutions of the form

$$
a_{6}(t)=\left(\alpha \cos 2 \sqrt{-\frac{\Lambda}{3}} t+\beta \sin 2 \sqrt{-\frac{\Lambda}{3}} t\right)^{\frac{1}{2}} .
$$

\section{Discussion and Conclusions}

To have a more complete presentation of the contents of this paper, some main considerations should be discussed. These considerations include the gained new cosmological solutions, used eigenvalue method and nonlocal operator.

On new cosmological solutions. Section 3 is related to the finding of new cosmological solutions of nonlocal gravity model (2). In a class of possible scale factors of the form $a(t)=\left(\alpha e^{\lambda t}+\beta e^{-\lambda t}\right)^{\gamma}$, we found four new solutions when $\gamma=1$ and no nontrivial solutions if $\gamma \neq 1$. The new solutions are

$$
\begin{aligned}
& a(t)=A e^{ \pm \sqrt{\frac{\Lambda}{3}} t}, \quad R(t)=\frac{6 k}{A^{2}} e^{\mp 2 \sqrt{\frac{\Lambda}{3}} t}+4 \Lambda, \quad k=+1,-1, \quad \Lambda>0 . \\
& a(t)=\frac{1}{\sqrt{\Lambda}} \cosh \left(\sqrt{\frac{\Lambda}{3}} t\right), \quad R(t)=8 k \Lambda \frac{1}{\cosh ^{2}\left(\sqrt{\frac{\Lambda}{3}} t\right)}+4 \Lambda, \quad k=-1, \quad \Lambda>0 . \\
& a(t)=\frac{1}{\sqrt{\Lambda}} \sinh \left(\sqrt{\frac{\Lambda}{3}} t\right), \quad R(t)=8 k \Lambda \frac{1}{\sinh ^{2}\left(\sqrt{\frac{\Lambda}{3}} t\right)}+4 \Lambda, \quad k=+1, \quad \Lambda>0 . \\
& a(t)=\frac{1}{\sqrt{-\Lambda}} \sin \left(\sqrt{\frac{-\Lambda}{3}} t\right), \quad R(t)=-8 k \Lambda \frac{1}{\sin ^{2}\left(\sqrt{\frac{-\Lambda}{3}} t\right)}+4 \Lambda, \quad k=+1, \quad \Lambda<0 .
\end{aligned}
$$


Recall that in the de Sitter (anti-de Sitter) (5) case, analogous solutions are

$$
\begin{aligned}
& a(t)=A e^{ \pm \sqrt{\frac{\Lambda}{3}}}, \quad R=4 \Lambda, \quad k=0, \quad \Lambda>0 . \\
& a(t)=\sqrt{\frac{3}{\Lambda}} \cosh \left(\sqrt{\frac{\Lambda}{3}} t\right), \quad R=4 \Lambda, \quad k=+1, \quad \Lambda>0 . \\
& a(t)=\sqrt{\frac{3}{\Lambda}} \sinh \left(\sqrt{\frac{\Lambda}{3}} t\right), \quad R=4 \Lambda, \quad k=-1, \quad \Lambda>0 . \\
& a(t)=\sqrt{\frac{-3}{\Lambda}} \sin \left(\sqrt{\frac{-\Lambda}{3}} t\right), \quad R=4 \Lambda, \quad k=-1, \quad \Lambda<0 .
\end{aligned}
$$

Comparing (90)-(93) with (94)-(97), we can note that for the same cosmological constant $\Lambda$, there are analogous scale factors with the same time dependence, but with different curvature constant $k$. This fact can be interpreted as change in topology in de Sitter (anti-de Sitter) space by the inclusion of the nonlocal term of the form $(R-4 \Lambda) \mathcal{F}(\square)(R-4 \Lambda)$; see (2). For example, exponential expansion (94) in a flat de Sitter universe remains exponential (90) by nonlocal transition into closed or open de Sitter space. We can also conclude that this kind of nonlocality changes the constant space-time curvature $(R=4 \Lambda)$ to the time-dependent one $(R=R(t))$. It is worth noting that in the nonlocal square root gravity model (3), there is a cosmological solution with the scale factor $a(t)=A e^{ \pm \sqrt{\frac{\Lambda}{6}} t}, \quad \Lambda>0, k=+1,-1$, with scalar curvature $R(t)=\frac{6 k}{A^{2}} e^{\mp \sqrt{\frac{2}{3} \Lambda} t}+2 \Lambda$; see Section 3.3 in [48]. This case is similar to (90) presented in this paper. We expect that analogous cases exist in some other examples of transition from the local to nonlocal de Sitter model.

On the eigenvalue method. In our approach, to solve equations of motion in the case of a homogeneous and isotropic universe, an essential role is the possibility to solve the corresponding eigenvalue problem $\square(R(t)-4 \Lambda)=q(R(t)-4 \Lambda)$, where $q=\zeta \Lambda$. $\Lambda$ appears here on the basis of dimensionality. Analogous solutions of (90)-(93) and (94)-(97) have the same Hubble parameter $H(t)=\frac{\dot{a}}{a}$ and, consequently, the same d'AlembertBeltrami operator $\square=-\frac{\partial^{2}}{\partial t^{2}}-3 H(t) \frac{\partial}{\partial t}$.

One can easily see that solution of $\square(R-4 \Lambda)=q(R-4 \Lambda)$ implies the solution of the following eigenvalue problem:

$$
\square^{-1}(R-4 \Lambda)=q^{-1}(R-4 \Lambda), \quad q \neq 0 .
$$

In other words, operators $\square$ and $\square^{-1}$ have the same eigenfunctions $R(t)-4 \Lambda$, but with different eigenvalues $q$ and $1 / q$, when $q \neq 0$.

On the nonlocal operator. The solvability of the eigenvalue problem (98) gives rise to introduce an extended version of the nonlocal operator $\mathcal{F}(\square)=\sum_{n=1}^{+\infty} f_{n} \square^{n}$ to the following one:

$$
\mathcal{F}(\square)=\sum_{n=-\infty}^{+\infty} f_{n} \square^{n}=\sum_{n=1}^{+\infty} f_{n} \square^{n}+f_{0}+\sum_{n=1}^{+\infty} f_{-n} \square^{-n},
$$

where $f_{0}=0$ in (2) nonlocal gravity model. Note that the nonlocal operator (99) is symmetric under interchange $n \longleftrightarrow-n$. This extended nonlocal operator satisfies the eigenvalue problem $\mathcal{F}(\square)(R-4 \Lambda)=\mathcal{F}(q)(R-4 \Lambda)$, where

$$
\mathcal{F}(q)=\sum_{n \neq 0} f_{n} q^{n}=\sum_{n=1}^{+\infty} f_{n} q^{n}+\sum_{n=1}^{+\infty} f_{-n} q^{-n} .
$$

In Section 3, we could replace $\mathcal{F}(\square)$ by this one in (99) with $f_{0}=0$, and the same new scale factors would be obtained with the same constraints on this extended $\mathcal{F}(\square)$. Note that now eigenvalues are $q=\frac{2}{3} \Lambda, \Lambda \neq 0$ and $q^{-1}=\frac{3}{2} \frac{1}{\Lambda}, \Lambda \neq 0$ for all four new solutions. 
Note that the finding of each new cosmological solution induces two restrictions on nonlocal operator $\mathcal{F}(\square)$. At this stage, an explicit form of $\mathcal{F}(\square)$ is not necessary.

On further investigations. The absence of the additional degrees of freedom, particularly ghosts, should be an important property of nonlocal gravity. A ghost-free condition is investigated in paper [43] for models of form (1), which includes our model (2); see also $[21,50]$ and the references therein. To avoid a ghost, nonlocal operator $\mathcal{F}$ must satisfy some conditions that depend on the background cosmological solution. This needs detailed investigation of the second variation [39] of action (1) and is a subject for future consideration.

As shown in Section 2, nonlocal gravity model (2) can be derived from nonlocal de Sitter gravity (3). These two models together contain cosmological solutions that mimic the interference of dark energy with radiation and dark matter in the flat universe. Both models also have a nonsingular bounce solution. Hence, at the cosmological scale, these nonlocal models imitate some effects that are a part of cosmic history described by standard model of cosmology ( $\Lambda \mathrm{CDM}$ model). This situation gives rise to continue with developments of this nonlocal gravity approach and explore the influence on astrophysical effects at the galactic scale and the solar system. In addition, the possible inflation, cosmic microwave background (CMB) and cosmological perturbations should be investigated [51].

Conclusions. At the end, it is worth noting the main results presented in this paper.

- Four new exact cosmological solutions are obtained.

- Effective energy density and effective pressure are computed for all new solutions.

- Change of space topology by nonlocal gravity is noted.

- A connection between nonlocal gravity models (2) and (3) is shown.

- The method of finding eigenfunctions $R(t)-4 \Lambda$ is further elaborated.

- Nonlocal operator $\mathcal{F}(\square)$ can be naturally extended by the addition of $\square^{-1}$ in a symmetric way.

Author Contributions: All authors have equally contributed to conceptualization and methodology, formal analysis and investigation, writing and editing of the manuscript. All authors have read and agreed to the published version of the manuscript.

Funding: This research was partially funded by the Ministry of Education, Science and Technological Developments of the Republic of Serbia, grant number 451-03-9/2021-14/200104.

Institutional Review Board Statement: Not applicable.

Informed Consent Statement: Not applicable.

Data Availability Statement: Not applicable.

Conflicts of Interest: The authors declare no conflict of interest.

\section{References}

1. Robson, B.A. Introductory chapter: Standard Model of Cosmology. In Redefining Standard Model Cosmology; Intechopen: London, UK, 2019; pp. 1-4. [CrossRef]

2. Wald, R.M. General Relativity; University of Chicago Press: Chicago, IL, USA, 1984.

3. Oks, E. Brief review of recent advances in understanding dark matter and dark energy. New Astron. Rev. $2021,93,101632$. [CrossRef]

4. Ellis, G.F.R. 100 years of general relativity. In General Relativity and Gravitation: A Centennial Perspective; Cambridge University Press: Cambridge, UK, 2015; pp. 10-19.

5. Sotiriou, T.P.; Faraoni, V. $f(R)$ theories of gravity. Rev. Mod. Phys. 2010, 82, 451-497. [CrossRef]

6. Clifton, T.; Ferreira, P.G.; Padilla, A.; Skordis, C. Modified gravity and cosmology. Phys. Rep. 2012, 513, 1-189. [CrossRef]

7. Nojiri, S.; Odintsov, S.D. Unified cosmic history in modified gravity: From $F(R)$ theory to Lorentz non-invariant models. Phys. Rep. 2011, 505, 59-144. [CrossRef]

8. Nojiri, S.; Odintsov, S.D.; Oikonomou, V.K. Modified gravity theories on a nutshell: Inflation, bounce and late-time evolution. Phys. Rep. 2017, 692, 1-104. [CrossRef]

9. Novello, M.; Bergliaffa, S.E.P. Bouncing cosmologies. Phys. Rep. 2008, 463, 127-213. [CrossRef]

10. Antoniadis, I.; Chatrabhuti, A. Challenges in supersymmetric cosmology. Symmetry 2020, 12, 468. [CrossRef] 
11. Benisty, D.; Guendelman, E.I.; Nissimov, E.; Pacheva, S. Dynamically generated inflationary $\Lambda$ CDM. Symmetry $2020,12,481$. [CrossRef]

12. Bilić, N.; Dimitrijević, D.D.; Djordjevic, G.S.; Milošević, M.; Stojanović, M. Tachyon inflation in the holographic braneworld. JCAP 2019, 8, 034. [CrossRef]

13. Deser, S.; Woodard, R. Nonlocal cosmology. Phys. Rev. Lett. 2007, 99, 111-301. [CrossRef]

14. Woodard, R.P. Nonlocal models of cosmic acceleration. arXiv 2014, arXiv:1401.0254.

15. Belgacem, E.; Dirian, Y.; Foffa, S.; Maggiore, M. Nonlocal gravity. Conceptual aspects and cosmological predictions. J. Cosmol. Astropart. Phys. 2018, 1803, 002. [CrossRef]

16. Biswas, T.; Mazumdar, A.; Siegel, W. Bouncing universes in string-inspired gravity. J. Cosmol. Astropart. Phys. 2006, 0603, 009. [CrossRef]

17. Biswas, T.; Koivisto, T.; Mazumdar, A. Towards a resolution of the cosmological singularity in non-local higher derivative theories of gravity. J. Cosmol. Astropart. Phys. 2010, 1011, 008. [CrossRef]

18. Biswas, T.; Gerwick, E.; Koivisto, T.; Mazumdar, A. Towards singularity and ghost free theories of gravity. Phys. Rev. Lett. 2012, 108, 031-101. [CrossRef] [PubMed]

19. Biswas, T.; Koshelev, A.S.; Mazumdar, A.; Vernov, S.Y. Stable bounce and inflation in non-local higher derivative cosmology. J. Cosmol. Astropart. Phys. 2012, 08, 024. [CrossRef]

20. Biswas, T.; Conroy, A.; Koshelev, A.S.; Mazumdar, A. Generalized gost-free quadratic curvature gravity. Class. Quantum Grav. 2014, 31, 159501. [CrossRef]

21. Biswas, T.; Koshelev, A.S.; Mazumdar, A. Consistent higher derivative gravitational theories with stable de Sitter and Anti-de Sitter backgrounds. Phys. Rev. 2017, D 95, 043533. [CrossRef]

22. Kolar, I.; Torralba, F.J.M.; Mazumdar, A. New non-singular cosmological solution of non-local gravity. arXiv 2021, arXiv:2109.02143.

23. Dragovich, B. On Nonlocal modified gravity and cosmology. Springer Proc. Math. Stat. 2014, 111, 251-262.

24. Koshelev, A.S.; Vernov, S.Y. On bouncing solutions in non-local gravity. Phys. Part. Nuclei 2012, 43, 666-668. [CrossRef]

25. Koshelev, A.S. Stable analytic bounce in non-local Einstein-Gauss-Bonnet cosmology. Class. Quantum Grav. $2013,30,155001$. [CrossRef]

26. Kumar, K.S. Non-local $D^{2}$-like inflation, gravitational waves and non-gaussianities. arXiv 2021, arXiv:2111.04291.

27. Koshelev, A.S.; Kumar, K.S.; Starobinsky, A.A. Analytic infinite derivative gravity, R2-like inflation, quantum gravity and CMB. Int. J. Mod. Phys. D 2020, 29, 2043018. [CrossRef]

28. Koshelev, A.S.; Kumar, K.S.; Starobinsky, A.A. $R^{2}$ inflation to probe non-perturbative quantum gravity. JHEP 2018, $1803,071$. [CrossRef]

29. Koshelev, A.S.; Modesto, L.; Rachwal, L.; Starobinsky, A.A. Occurrence of exact $R^{2}$ inflation in non-local UV-complete gravity. JHEP 2016, 11, 1-41.

30. Buoninfante, L.; Koshelev, A.S.; Lambiase, G.; Mazumdar, A. Classical properties of non-local, ghost- and singularity-free gravity. JCAP 2018, 1809, 034. [CrossRef]

31. Koshelev, A.S.; Marto, J.; Mazumdar, A. Towards conformally flat, non-Kasner vacuum solution in infinite derivative gravity. JCAP 2019, 1902, 020. [CrossRef]

32. Elizalde, E.; Pozdeeva, E.O.; Vernov, S.Y. Stability of de Sitter solutions in non-local cosmological models. PoS 2012, $138,038$.

33. Conroy, A.; Koivisto, T.; Mazumdar, A.; Teimouri, A. Generalised quadratic curvature, non-local infrared modifications of gravity and Newtonian potentials. Clas. Quantum Grav. 2015, 32, 015024. [CrossRef]

34. Dialektopoulos, K.F.; Borka, D.; Capozziello, S.; Borka Jovanović, V.; Jovanović, P. Constraining nonlocal gravity by S2 star orbits. Phys. Rev. D 2019, 99, 044053. [CrossRef]

35. Dragovich, B.; Khrennikov, A.Y.; Kozyrev, S.V.; Volovich, I.V.; Zelenov, E.I. $p$-Adic mathematical physics: The first 30 years. $p$-Adic Numbers Ultrametric Anal. Appl. 2017, 9, 87-121. [CrossRef]

36. Stelle, K.S. Renormalization of higher derivative quantum gravity. Phys. Rev. D 1977, 16, 953. [CrossRef]

37. Modesto, L. Super-renormalizable quantum gravity. Phys. Rev. D 2012, 86, 044005. [CrossRef]

38. Modesto, L.; Rachwal, L. Super-renormalizable and finite gravitational theories. Nucl. Phys. B 2014, 889, 228. [CrossRef]

39. Dimitrijevic, I.; Dragovich, B.; Rakic, Z.; Stankovic, J. Variations of infinite derivative modified gravity. Springer Proc. Math. Stat. 2018, 263, 91-111.

40. Dimitrijevic, I.; Dragovich, B.; Grujic, J.; Rakic, Z. On modified gravity. Springer Proc. Math. Stat. 2013, 36, $251-259$.

41. Dimitrijevic, I.; Dragovich, B.; Grujic, J.; Rakic, Z. New cosmological solutions in nonlocal modified gravity. Rom. J. Phys. 2013, 58, 550-559.

42. Dimitrijevic, I.; Dragovich, B.; Grujic, J.; Rakic, Z. Some power-law cosmological solutions in nonlocal modified gravity. Springer Proc. Math. Stat. 2014, 111, 241-250.

43. Dimitrijevic, I.; Dragovich, B.; Grujic, J.; Koshelev, A.S.; Rakic, Z. Cosmology of non-local $f(R)$ gravity. Filomat 2019, 33, $1163-1178$. [CrossRef]

44. Dimitrijevic, I.; Dragovich, B.; Stankovic, J.; Koshelev, A.S.; Rakic, Z. On nonlocal modified gravity and its cosmological solutions. Springer Proc. Math. Stat. 2016, 191, 35-51. 
45. Dimitrijevic, I.; Dragovich, B.; Grujic, J.; Rakic, Z. Some cosmological solutions of a nonlocal modified gravity. Filomat 2015, 29, 619-628. [CrossRef]

46. Dimitrijevic, I. Cosmological solutions in modified gravity with monomial nonlocality. Appl. Math. Comput. 2016, 285, 195-203. [CrossRef]

47. Dimitrijevic, I.; Dragovich, B.; Rakic, Z.; Stankovic, J. On nonlocal gravity with constant scalar curvature. Publ. Inst. Math. 2018, 103, 53-59. [CrossRef]

48. Dimitrijevic, I.; Dragovich, B.; Koshelev, A.S.; Rakic, Z.; Stankovic, J. Cosmological solutions of a nonlocal square root gravity. Phys. Lett. B 2019, 797, 134848. [CrossRef]

49. Dimitrijevic, I.; Dragovich, B.; Koshelev, A.S.; Rakic, Z.; Stankovic, J. Some cosmological solutions of a new nonlocal gravity model. Symmetry 2020, 12, 917. [CrossRef]

50. Buoninfante, L.; Lambiase, G.; Miyashita, Y.; Takebe, W.; Yamaguchi, M. Generalized ghost-free propagators in nonlocal field theories. Phys. Rev. D 2020, 101, 084019. [CrossRef]

51. Mukhanov, V. Physical Foundations of Cosmology; Cambridge University Press: Cambridge, UK, 2005. 